\title{
Risk factors for suicide in bipolar disorder: a cohort study of 12850 patients
}

Hansson C, Joas E, Pålsson E, Hawton K, Runeson B, Landén M. Risk factors for suicide in bipolar disorder: a cohort study of 12850 patients.

Objective: Bipolar disorder carries a high risk of suicide. Identification of risk factors is important. The aim of this study was to study risk factors for suicide in a large cohort of men and women with bipolar disorder.

Method: A prospective cohort study using clinical data from the Swedish National Quality Register for Bipolar Affective Disorder (BipoläR). The outcome variable was suicide captured in the Cause of Death Register between 2004 and 2014. Hazard ratios (HR) were calculated using Cox proportional hazards models.

Results: Of 12850 persons (4844 men and 8006 women) with bipolar disorder, 90 ( 55 men and 35 women) died by suicide during the followup period (between 1 and 10 years). Male sex (HR 2.56), living alone (HR 2.45), previous suicide attempts (HR 4.10), comorbid psychiatric disorder (HR 2.64), recent affective episodes (HR 2.39), criminal conviction (HR 4.43), psychiatric inpatient care (HR 2.79), and involuntary commitment (HR 3.50) were significant risk factors for suicide. Several of the statistically significant risk factors for suicide in bipolar disorder differed between men and women.

Conclusions: Risk factors for suicide in bipolar disorder include factors associated with suicide in general, but also diagnosis-specific factors.

\author{
C. Hansson ${ }^{1}$ (DD, E. Joas ${ }^{1}$, \\ E. Pålsson ${ }^{1}$, K. Hawtonn ${ }^{2}$ \\ B. Runeson ${ }^{3}$, M. Landén ${ }^{1,4}$ \\ ${ }^{1}$ Department of Psychiatry and Neurochemistry, Institute \\ of Neuroscience and Physiology, Sahlgrenska Academy, \\ University of Gothenburg, Gothenburg, Sweden, ${ }^{2}$ Centre \\ for Suicide Research, University Department of \\ Psychiatry, Oxford, UK, ${ }^{3}$ Department of Clinical \\ Neuroscience, Center for Psychiatry Research and \\ ${ }^{4}$ Department of Medical Epidemiology and Biostatistics, \\ Karolinska Institutet, Stockholm, Sweden
}

\footnotetext{
This is an open access article under the terms of the Creative Commons Attribution-NonCommercial-NoDerivs License, which permits use and distribution in any medium, provided the original work is properly cited, the use is non-commercial and no modifications or adaptations are made.

Key words: bipolar disorder; suicide; risk factors Caroline Hansson, Department of Psychiatry and Neurochemistry, Institute of Neuroscience and Physiology, University of Gothenburg, Gothenburg Sweden.

E-mail: caroline.hansson@gu.se

Accepted for publication July 12, 2018
}

\section{Significant outcomes}

- Risk factors for suicide in bipolar disorder include not only general risk factors but also recent mood episodes that are specific for bipolar disorder.

- Bipolar subtype was not associated with suicide.

- Risk factors for suicide differ between men and women with bipolar disorder.

\section{Limitations}

- Although the study is large, the cohort does not include all patients with bipolar disorder in Sweden.

- The results of this study from Sweden may not be generalizable to other countries.

\section{Introduction}

Suicide is the 15th leading cause of death worldwide, with around 800000 annual suicides worldwide (1) and 1500 annual suicides in Sweden (2). It has been estimated that $90 \%$ of all suicide victims suffer from a psychiatric disorder (3). Individuals with bipolar affective disorder constitute a high-risk group with an estimated risk of suicide that is $17-20$ times higher than in the general population (4-6). A number of risk factors generally associated with suicide have been identified. But it is also important to determine risk factors for specific populations, e.g., in specific psychiatric disorders, as these might differ - or have different weight - across diagnoses (4). 
A systematic review identified male sex, a history of attempted suicide and expressed hopelessness as risk factors for suicide in bipolar disorder (7). However, the sample sizes of included studies were small - the number of suicides ranged from 3 to 18 - and only four of the studies had a prospective study design.

The Swedish National Quality Register for Bipolar Affective Disorder (BipoläR) contains clinical and demographic data for a large number of patients and is well suited for assessing clinical risk factors. Patients are followed up annually, allowing for the prospective study design needed to identify predictors of suicide. We have previously used BipoläR to study risk factors for attempted suicide in bipolar disorder. We found that the most important risk factors for attempted suicide were recent affective episodes, previous suicide attempts, and recent psychiatric inpatient care (8). Risk factors for suicide attempts may, however, differ from risk factors for suicide (9).

\section{Aims of the study}

The aim was to investigate risk factors for completed suicide in men and women with bipolar disorder in a prospective cohort study of 12850 individuals with bipolar disorder.

\section{Material and methods}

Participants

The study was designed as a longitudinal cohort study. The cohort of patients was identified from the Swedish National Quality Register for Bipolar Affective Disorder (BipoläR), which was established in 2004 with the aim of improving the quality of care for persons with bipolar disorder in Sweden. Sweden has more than 100 quality registers for various disorders and medical procedures. For an overview, see (10). All patients with bipolar disorder who receive treatment at psychiatric outpatient clinics in Sweden are possible to register and follow in BipoläR. According to Swedish law, registration in Swedish quality registers follow an opt-out procedure where patients must be informed that data are recorded but may decline to participate, in which case data cannot be recorded. De-identified data may be used for research purposes provided that the research project has been approved by the ethical review board.

All patients that entered in the Bipolä $\mathrm{R}$ register between 2004 and 2013 were included in the study. BipoläR contains individualized data on diagnoses (i.e., bipolar disorder type 1 (DSM-codes 296.1-
296.7), type 2 (296.89), not otherwise specified [NOS] (296.80), or schizoaffective disorder of bipolar type (295.70), along with comorbid psychiatric conditions, interventions, and outcomes. The register also captures basic clinical epidemiological data as well as longitudinal data on the natural history and clinical course of the disease. Data are collected by psychiatrists and staff managing the care of patients and thus have access to all clinical data. Diagnoses in BipoläR are made by the treating clinician according to DSM-IV-TR. Data from the quality register show that formal use of structured psychiatric diagnostic instruments (e.g., SCID or M.I.N.I. psychiatric interview) was employed in $47 \%$ of the cases.

BipoläR contains much more detailed phenotypic information than Swedish national population registers and the data quality is considered to be high (11). At the baseline registration - that can occur at any point during the course of illness - the following are documented: main psychiatric diagnosis and comorbidities, family history of affective disorder, age at onset, psychosocial and environmental problem/s (axis IV of the DSM-IV multiaxial system subdivided on family-, occupational-, or economy-related problems), violent behavior, suicide attempts, medical interventions, the number of affective episodes during the past 12 months, psychiatric inpatient care during the past 12 months, involuntary commitment during the past 12 months, criminal conviction during the past 12 months, sociodemographic variables, weight, and height, and ratings according to the Clinical Global Impression (CGI) and the Global Assessment of Functioning (GAF) rating scales. The same variables are documented at the annual follow-up registrations.

\section{Potential risk factors}

The following variables were tested for their association with suicide: sex, age, body mass index (BMI), education (completed higher education), living alone, psychosocial and environmental problem $/ \mathrm{s}$ (axis IV of the DSM-IV multiaxial system: family-, occupational, or economy-related problems), violent behavior (directed toward other people), criminal conviction in the previous year, bipolar disorder subtype (type 1, type 2, not otherwise specified, and schizoaffective disorder of bipolar type), affective episode/s (depressive, hypomanic, manic, or mixed) in the previous year, family history of affective disorder (bipolar disorder, unipolar disorder, or dysthymia in first-degree relatives), age at onset of any psychiatric disorder (before or after 18 years of age), any comorbid psychiatric disorder/s, comorbid substance use disorder, comorbid anxiety 


\section{Hansson et al.}

disorder, comorbid eating disorder, comorbid personality disorder, previous suicide attempt/s, psychiatric inpatient care in the previous year, and involuntary commitment in the previous year. According to the Compulsory Psychiatric Care Act in Sweden, the following criteria must be fulfilled: (i) the person suffers from a severe mental disorder, (ii) the person has an indispensable need for inpatient psychiatric care, and (iii) the person opposes voluntary care. The quality register provided two versions of data entry forms: one full version and one abbreviated version. Participating care units could use which one they preferred to use. For this reason, the $\mathrm{N}$ varies across variables.

\section{Identification of deaths by suicide}

The outcome variable was suicide registered in the Cause of Death Register between 2004 and 2014. The Cause of Death Register provides mortality data from more than $99 \%$ of all deaths occurring in Sweden (12). In Sweden, the death of a person is verified by a medical doctor who also establishes the cause of death. In consistence with previous research (13) and to avoid underestimation of suicides (14), suicide was classified according to ICD10 codes as either definite suicide (X60-84) or death by self-harm with undetermined intent (Y10-34).

\section{Statistical analyses}

We used Cox models with time-dependent covariates to analyze the association between different exposures and suicide (15). The hazard ratios were adjusted for age and sex with the exception of those that tested risk factors within sexes, which were adjusted for age only. The start time was set as the date of first entry into the register. Covariates, if time-varying, were updated at each new registration. End of follow-up was either date of suicide, date of other causes of death, or 31 December 2014. In addition, questions regarding the number of specific episodes in the previous year were only asked in follow-up questionnaires, therefore only individuals who had at least one followup were entered into these analyses, with start of follow-up time set at the first follow-up registration. Results are reported as Hazard Ratios (HR) with $95 \%$ confidence intervals. The statistical software $\mathrm{R}$ version 3.4 .3 was used for all analyses.

\section{Ethics}

The study was approved by the Gothenburg Regional Ethics Committee (294-11), Gothenburg, Sweden.

\section{Results}

We identified 12850 persons (4844 men and 8006 women) with bipolar disorder in BipoläR, of whom 90 (55 men and 35 women) died by suicide during the follow-up period up to the end of 2014. The follow-up time ranged from 1 year to 10 years, the mean follow-up time was 4.05 years, the median follow-up time was 3.80 years, and the mean number of registrations was 2.45 registrations per person. Clinical characteristics of the overall cohort are presented in Table 1. The numbers of patients having each potential risk factor at least once during the study period are presented in Table S1.

Table 1. Clinical characteristics of the study cohort at first registration stratified on patients that died by suicide during the study period and those that did not

\begin{tabular}{|c|c|c|}
\hline & Suicide & No suicide \\
\hline \multicolumn{3}{|l|}{ Sex, $n(\%)$} \\
\hline Men & $55(61.1)$ & $4789(37.5$ \\
\hline Women & $35(38.9)$ & $7971(62.5$ \\
\hline Age, mean (SD) & $47.4(14.8)$ & $47.8(16.0$ \\
\hline \multicolumn{3}{|l|}{ Bipolar disorder type, $n(\%)$} \\
\hline Type I & $45(50.0)$ & $5091(39.9$ \\
\hline Type II & $31(34.4)$ & $4953(38.8$ \\
\hline NOS & $12(13.3)$ & $2364(18.5$ \\
\hline SADB & $2(2.2)$ & $350(2.7)$ \\
\hline Missing data & $0(0.0)$ & $2(0.0)$ \\
\hline \multicolumn{3}{|l|}{ Family history of affective disorder, $n(\%)$} \\
\hline Yes & $23(25.6)$ & $3242(25.4$ \\
\hline No & $39(43.3)$ & $4598(36.0$ \\
\hline Missing data & $28(31.1)$ & $4920(38.6$ \\
\hline \multicolumn{3}{|l|}{ Early onset of psychiatric symptoms (<18 years), $n(\%)$} \\
\hline Yes & $21(23.3)$ & $2725(21.4$ \\
\hline No & $33(36.7)$ & $3913(30.7$ \\
\hline Missing data & $36(40.0)$ & $6122(48.0$ \\
\hline \multicolumn{3}{|l|}{ Education, $n(\%)$} \\
\hline Not completed elementary school & $6(6.7)$ & $739(5.8)$ \\
\hline Completed elementary school & $11(12.2)$ & $2283(17.9$ \\
\hline Completed high school & $37(41.1)$ & $4412(34.6$ \\
\hline At least 2 years higher education (university level) & $33(36.7)$ & $4270(33.5$ \\
\hline Missing data & $3(3.3)$ & $1056(8.3)$ \\
\hline \multicolumn{3}{|l|}{ Lithium, $n(\%)$} \\
\hline Yes & $56(62.2)$ & $7164(56.1$ \\
\hline No & $34(37.8)$ & $5570(43.7$ \\
\hline Missing data & $0(0.0)$ & $26(0.2)$ \\
\hline \multicolumn{3}{|l|}{ Valproate, $n(\%)$} \\
\hline Yes & $9(10.0)$ & $1215(9.5)$ \\
\hline No & $81(90.0)$ & $11519(90.3$ \\
\hline Missing data & $0(0.0)$ & $26(0.2)$ \\
\hline \multicolumn{3}{|l|}{ Lamotrigine, $n(\%)$} \\
\hline Yes & $20(22.2)$ & $3124(24.5$ \\
\hline No & $70(77.8)$ & $9610(75.3$ \\
\hline Missing data & $0(0.0)$ & $26(0.2)$ \\
\hline \multicolumn{3}{|l|}{ Antidepressants, $n(\%)$} \\
\hline Yes & $51(56.7)$ & $5286(41.4$ \\
\hline No & $36(40.0)$ & $6674(52.3$ \\
\hline Missing data & $3(3.3)$ & $800(6.3)$ \\
\hline \multicolumn{3}{|l|}{ Antipsychotics, $n(\%)$} \\
\hline Yes & $41(45.6)$ & $4310(33.8$ \\
\hline No & $46(51.1)$ & $7650(60.0$ \\
\hline Missing data & $3(3.3)$ & $800(6.3)$ \\
\hline
\end{tabular}

NOS, not otherwise specified; SADB, schizoaffective order, bipolar (manic) type. 
Risk factors for suicide in bipolar disorder

Table 2. Potential risk factors for suicide in bipolar disorder patients

\begin{tabular}{|c|c|c|c|c|c|c|c|c|c|c|}
\hline & $N$ & $N_{\text {suicides }}$ & $\mathrm{HR}$ & \multicolumn{2}{|c|}{$(95 \% \mathrm{Cl})$} & $P$ & $\mathrm{HR}_{\mathrm{adj}^{*}}$ & \multicolumn{2}{|c|}{$(95 \% \mathrm{Cl})_{\text {adj* }}$} & $P_{\text {adj* }}$ \\
\hline \multicolumn{11}{|l|}{ Sociodemographic and anthropometric factors } \\
\hline Male sex & 12850 & 90 & 2.53 & 1.66 & 3.87 & $<0.01$ & 2.56 & 1.68 & 3.92 & $<0.01$ \\
\hline Age & 12850 & 90 & 1.00 & 0.98 & 1.01 & 0.66 & 0.99 & 0.98 & 1.01 & 0.45 \\
\hline BMl & 12287 & 86 & 1.00 & 0.96 & 1.04 & 0.87 & 1.00 & 0.96 & 1.05 & 0.90 \\
\hline Education (completed higher education) & 11791 & 87 & 0.98 & 0.63 & 1.51 & 0.92 & 0.98 & 0.63 & 1.52 & 0.93 \\
\hline Living alone & 7138 & 51 & 2.44 & 1.35 & 4.42 & $<0.01$ & 2.45 & 1.36 & 4.43 & $<0.01$ \\
\hline Complicating social factors & 11825 & 85 & 1.23 & 0.78 & 1.92 & 0.37 & 1.27 & 0.81 & 2.01 & 0.30 \\
\hline Violent behavior & 6921 & 51 & 1.88 & 1.00 & 3.54 & 0.05 & 1.55 & 0.81 & 2.97 & 0.18 \\
\hline Criminal conviction & 7083 & 51 & 5.87 & 1.83 & 18.85 & $<0.01$ & 4.43 & 1.35 & 14.53 & 0.01 \\
\hline \multicolumn{11}{|l|}{ Disease-related factors } \\
\hline Bipolar disorder type & 12849 & 90 & & & & & & & & \\
\hline Type 1 (ref) & & & 1 & & & & & & & \\
\hline Type 2 & & & 0.74 & 0.46 & 1.18 & 0.21 & 0.78 & 0.49 & 1.25 & 0.31 \\
\hline NOS & & & 0.72 & 0.39 & 1.31 & 0.28 & 0.75 & 0.41 & 1.36 & 0.34 \\
\hline SADB & & & 0.55 & 0.13 & 2.25 & 0.40 & 0.55 & 0.13 & 2.27 & 0.41 \\
\hline Any affective episode in the previous year & 12436 & 90 & 2.23 & 1.38 & 3.59 & $<0.01$ & 2.39 & 1.47 & 3.88 & $<0.01$ \\
\hline Any depressive episode in the previous year & 6631 & 48 & 2.17 & 1.22 & 3.88 & 0.01 & 2.24 & 1.25 & 4.01 & 0.01 \\
\hline Any (hypo-)manic episode in the previous year & 6632 & 48 & 0.91 & 0.47 & 1.79 & 0.79 & 0.88 & 0.44 & 1.73 & 0.71 \\
\hline Any mixed episode in the previous year & 6628 & 48 & 1.31 & 0.52 & 3.32 & 0.57 & 1.33 & 0.52 & 3.40 & 0.55 \\
\hline Family history of affective disorder & 7902 & 62 & 0.72 & 0.43 & 1.20 & 0.21 & 0.74 & 0.44 & 1.24 & 0.26 \\
\hline Early onset of psychiatric problems (<18 years) & 6692 & 54 & 0.95 & 0.55 & 1.64 & 0.85 & 0.92 & 0.50 & 1.68 & 0.78 \\
\hline Any comorbid psychiatric disorder & 11802 & 83 & 2.46 & 1.60 & 3.78 & $<0.01$ & 2.64 & 1.69 & 4.13 & $<0.01$ \\
\hline Comorbid substance use disorder & 11802 & 83 & 4.50 & 2.64 & 7.67 & $<0.01$ & 3.79 & 2.21 & 6.50 & $<0.01$ \\
\hline Comorbid anxiety disorder & 11802 & 83 & 1.74 & 0.98 & 3.09 & 0.06 & 1.91 & 1.07 & 3.42 & 0.03 \\
\hline Comorbid eating disorder & 11802 & 83 & 1.17 & 0.29 & 4.74 & 0.83 & 1.60 & 0.39 & 6.67 & 0.52 \\
\hline Comorbid personality disorder & 11802 & 83 & 2.15 & 0.93 & 4.92 & 0.07 & 2.49 & 1.07 & 5.77 & 0.03 \\
\hline Previous suicide attempt & 11747 & 84 & 3.30 & 2.01 & 5.41 & $<0.01$ & 4.10 & 2.43 & 6.92 & $<0.01$ \\
\hline Psychiatric inpatient care & 10106 & 63 & 2.84 & 1.66 & 4.87 & $<0.01$ & 2.79 & 1.62 & 4.80 & $<0.01$ \\
\hline Involuntary commitment & 9309 & 60 & 3.74 & 1.84 & 7.60 & $<0.01$ & 3.50 & 1.71 & 7.15 & $<0.01$ \\
\hline
\end{tabular}

HR, hazard ratio; BMI, body mass index; NOS, not otherwise specified; SADB, schizoaffective order, bipolar (manic) type.

${ }^{*}$ Adjusted for sex and age.

Table 2 displays associations between covariates and suicide using Cox proportional hazards models, both unadjusted and adjusted for sex and age. The statistically significant risk factors for completed suicide were: male sex (HR 2.56); living alone (HR 2.45); previous suicide attempts (HR 4.10); any comorbid psychiatric disorder (HR 2.64); comorbid substance use disorder (HR 3.79), anxiety disorder (HR 1.91), and personality disorder (HR 2.49); recent affective episode(s) (HR 2.39); recent depressive episode(s) (HR 2.24); criminal conviction (HR 4.43); psychiatric inpatient care (HR 2.79); and involuntary commitment (HR 3.50).

When analyzing the data for men and women separately, we found that living alone (HR 2.71), comorbid substance use disorder (HR 4.20), involuntary commitment (HR 4.30), and having had at least one affective episode in the previous year (HR 3.19) were significant predictors of suicide in men, but not in women. Conversely, we found that criminal conviction (HR 9.85), comorbid personality disorder (HR 4.78), and having had at least one depressive episode in the previous year (HR 2.81) were significant predictors of suicide in women, but not in men (Tables 3 and 4).

\section{Discussion}

In a prospective study of 12850 patients with bipolar disorder identified through a national Swedish quality assurance register for bipolar affective disorder, we identified 90 suicides that occurred during the follow-up period. In the whole cohort, the major risk factors for suicide were male sex, comorbid psychiatric illness - in particular substance use disorder, anxiety disorder, and personality disorder - previous suicide attempts, living alone, recent affective episodes, recent psychiatric inpatient care, recent involuntary commitment, and recent criminal conviction. We also found that several risk factors were statistically significant for men but not for women and vice versa.

Psychiatric disorders - substance use disorder, eating disorder, personality disorder, anxiety and depression - are common in people who die by suicide. Other established risk factors for suicide in general include male sex, previous suicide attempts, family history of suicide, and exposure to early life adversities (16). Some of these general risk factors for suicide have also been identified as risk factors for people with bipolar disorder, such as male sex, previous suicide attempts, family 


\section{Hansson et al.}

Table 3. Potential risk factors for suicide in male bipolar disorder patients

\begin{tabular}{|c|c|c|c|c|c|c|c|c|c|c|}
\hline & $N$ & $N_{\text {suicides }}$ & $\mathrm{HR}$ & \multicolumn{2}{|c|}{ (95\% Cl) } & $P$ & $\mathrm{HR}_{\mathrm{adj}^{*}}$ & \multicolumn{2}{|c|}{$(95 \% \mathrm{Cl})_{\text {adj* }}$} & $P_{\text {adj* }}$ \\
\hline \multicolumn{11}{|l|}{ Sociodemographic and anthropometric factors } \\
\hline Men & 4844 & 55 & & & & & & & & \\
\hline Age & 4844 & 55 & 0.99 & 0.98 & 1.01 & 0.42 & 0.99 & 0.98 & 1.01 & 0.42 \\
\hline $\mathrm{BMI}$ & 4635 & 52 & 1.00 & 0.94 & 1.06 & 0.93 & 1.00 & 0.94 & 1.06 & 0.95 \\
\hline Education (completed higher education) & 4404 & 52 & 0.96 & 0.55 & 1.68 & 0.89 & 0.99 & 0.56 & 1.75 & 0.98 \\
\hline Living alone & 2694 & 31 & 2.70 & 1.24 & 5.87 & 0.01 & 2.71 & 1.25 & 5.89 & 0.01 \\
\hline Violent behavior & 2602 & 31 & 1.45 & 0.67 & 3.14 & 0.35 & 1.36 & 0.62 & 3.00 & 0.45 \\
\hline Criminal conviction & 2676 & 31 & 3.61 & 0.86 & 15.17 & 0.08 & 3.30 & 0.77 & 14.07 & 0.11 \\
\hline \multicolumn{11}{|l|}{ Disease-related factors } \\
\hline Bipolar disorder type & 4844 & 55 & & & & & & & & \\
\hline Type 1 (ref) & & & 1 & & & & & & & \\
\hline Type 2 & & & 0.62 & 0.33 & 1.16 & 0.13 & 0.61 & 0.33 & 1.15 & 0.12 \\
\hline NOS & & & 0.57 & 0.25 & 1.29 & 0.18 & 0.56 & 0.25 & 1.28 & 0.17 \\
\hline Any affective episode in the previous year & 4670 & 55 & 3.20 & 1.68 & 6.08 & $<0.01$ & 3.19 & 1.66 & 6.14 & $<0.01$ \\
\hline Any depressive episode in the previous year & 2577 & 29 & 2.08 & 1.00 & 4.33 & 0.05 & 1.97 & 0.94 & 4.11 & 0.07 \\
\hline Any (hypo-)manic episode in the previous year & 2577 & 29 & 0.53 & 0.18 & 1.51 & 0.23 & 0.47 & 0.16 & 1.37 & 0.17 \\
\hline Any mixed episode in the previous year & 2575 & 29 & 1.72 & 0.52 & 5.71 & 0.37 & 1.56 & 0.47 & 5.20 & 0.47 \\
\hline Family history of affective disorder & 2982 & 38 & 0.59 & 0.30 & 1.18 & 0.14 & 0.60 & 0.30 & 1.19 & 0.14 \\
\hline Early onset of psychiatric problems (<18 years) & 2501 & 34 & 0.74 & 0.35 & 1.55 & 0.42 & 0.61 & 0.27 & 1.37 & 0.23 \\
\hline Any comorbid psychiatric disorder & 4447 & 52 & 2.55 & 1.48 & 4.39 & $<0.01$ & 2.57 & 1.46 & 4.52 & $<0.01$ \\
\hline Comorbid substance use disorder & 4447 & 52 & 4.23 & 2.29 & 7.80 & $<0.01$ & 4.20 & 2.28 & 7.75 & $<0.01$ \\
\hline Comorbid anxiety disorder & 4447 & 52 & 2.01 & 0.94 & 4.27 & 0.07 & 1.97 & 0.92 & 4.20 & 0.08 \\
\hline Comorbid eating disorder & 4447 & 52 & - & - & - & - & - & - & - & - \\
\hline Comorbid personality disorder & 4447 & 52 & 0.83 & 0.11 & 5.99 & 0.85 & 0.80 & 0.11 & 5.83 & 0.83 \\
\hline Previous suicide attempt & 4419 & 53 & 4.24 & 2.18 & 8.25 & $<0.01$ & 4.12 & 2.08 & 8.15 & $<0.01$ \\
\hline Psychiatric inpatient care & 3838 & 38 & 3.13 & 1.58 & 6.22 & $<0.01$ & 2.95 & 1.47 & 5.90 & $<0.01$ \\
\hline Involuntary commitment & 3537 & 37 & 4.65 & 2.04 & 10.60 & $<0.01$ & 4.30 & 1.87 & 9.86 & $<0.01$ \\
\hline
\end{tabular}

${ }^{*}$ Adjusted for age. HR, hazard ratio; BMI, body mass index; NOS, not otherwise specified; SADB, schizoaffective order, bipolar (manic) type.

history of suicide, and psychiatric comorbidity (17). In line with previous studies, we found that male sex, previous suicide attempts, and psychiatric comorbidities were significantly associated with suicide. Drilling deeper into the type of psychiatric comorbidity, we found that substance use disorder, personality disorder, and anxiety disorder was associated with suicide, while eating disorder was not. Interestingly, comorbid substance use disorder was strongly associated with suicide in men, but not statistically significant in women. This is noteworthy given previous inconsistent results: Whereas some studies have found an increased risk of suicide in bipolar disorder with comorbid substance use disorder in comparison to bipolar disorder without comorbid substance use disorder (18, 19), a meta-analysis only found a non-significant trend toward a positive association (odds ratio 1.20, 95\% CI: 0.93-1.56) (9). In line with our results, a study of bipolar disorder suicide victims showed that comorbid alcohol dependence was significantly more common in men than in women (20). Conversely, we found that comorbid personality disorder was a significant predictor of suicide in women but not in men. A previous study found that personality disorder was - along with alcohol dependence - the most common secondary diagnoses in persons with bipolar disorder who die by suicide (21). Another study found that personality disorder was associated with increased risk of suicide after a suicide attempt (22). Finally, we found that comorbid anxiety disorder was a risk factor for suicide, which is consistent with previous studies $(17,23,24)$.

Living alone, criminal conviction and recent inpatient care are also risk factors for suicide in general (16) that we could corroborate for bipolar disorder. We further found that living alone was a robust risk factor for suicide in men with bipolar disorder, but not in women. Living alone has previously been linked to suicide in bipolar disorder (25), but a meta-analysis showed no significant association between suicide in individuals with bipolar disorder and civil status, or living with family (7). We also found that criminal conviction was associated with suicide in women but not men with bipolar disorder. Criminal behavior has previously been reported to be associated with suicide in general $(26,27)$. Moreover, suicide rates are high in prisoners, especially prisoners with psychiatric disorders $(28,29)$, also after release from prison (30). Recent psychiatric inpatient care, which 
Risk factors for suicide in bipolar disorder

Table 4. Potential risk factors for suicide in female bipolar disorder patients

\begin{tabular}{|c|c|c|c|c|c|c|c|c|c|c|}
\hline & $N$ & $N_{\text {suicides }}$ & $\mathrm{HR}$ & \multicolumn{2}{|c|}{$(95 \% \mathrm{Cl})$} & $P$ & $\mathrm{HR}_{\mathrm{adj} ;}$ & \multicolumn{2}{|c|}{$(95 \% \mathrm{Cl})_{\mathrm{adj}}{ }^{*}$} & $P_{\text {adj* }}$ \\
\hline \multicolumn{11}{|l|}{ Sociodemographic and anthropometric factors } \\
\hline Women & 8006 & 35 & & & & & & & & \\
\hline Age & 8006 & 35 & 1.00 & 0.98 & 1.02 & 0.82 & 1.00 & 0.98 & 1.02 & 0.82 \\
\hline BMl & 7652 & 34 & 1.01 & 0.95 & 1.07 & 0.85 & 1.01 & 0.95 & 1.07 & 0.83 \\
\hline Education (completed higher education) & 7387 & 35 & 0.97 & 0.49 & 1.92 & 0.93 & 0.97 & 0.49 & 1.93 & 0.93 \\
\hline Living alone & 4444 & 20 & 2.08 & 0.83 & 5.22 & 0.12 & 2.08 & 0.83 & 5.22 & 0.12 \\
\hline Complicating social factors & 7362 & 31 & 1.05 & 0.50 & 2.24 & 0.89 & 1.07 & 0.50 & 2.30 & 0.86 \\
\hline Violent behavior & 4319 & 20 & 1.96 & 0.66 & 5.87 & 0.23 & 2.05 & 0.67 & 6.29 & 0.21 \\
\hline Criminal conviction & 4407 & 20 & 9.51 & 1.27 & 71.07 & 0.03 & 9.85 & 1.30 & 74.75 & 0.03 \\
\hline \multicolumn{11}{|l|}{ Disease-related factors } \\
\hline Bipolar disorder type & 8005 & 35 & & & & & & & & \\
\hline Type 1 (ref) & & & 1 & & & & & & & \\
\hline Type 2 & & & 1.20 & 0.56 & 2.56 & 0.63 & 1.19 & 0.55 & 2.56 & 0.65 \\
\hline NOS & & & 1.20 & 0.48 & 3.00 & 0.70 & 1.19 & 0.47 & 3.00 & 0.71 \\
\hline SADB & & & 0.94 & 0.12 & 7.16 & 0.95 & 0.93 & 0.12 & 7.15 & 0.95 \\
\hline Any affective episode in the previous year & 7766 & 35 & 1.55 & 0.76 & 3.18 & 0.23 & 1.56 & 0.75 & 3.24 & 0.24 \\
\hline Any depressive episode in the previous year & 4054 & 19 & 2.80 & 1.06 & 7.37 & 0.04 & 2.81 & 1.06 & 7.47 & 0.04 \\
\hline Any (hypo-)manic episode in the previous year & 4055 & 19 & 1.70 & 0.67 & 4.33 & 0.26 & 1.69 & 0.66 & 4.35 & 0.27 \\
\hline Any mixed episode in the previous year & 4053 & 19 & 1.15 & 0.26 & 4.97 & 0.85 & 1.12 & 0.25 & 4.95 & 0.88 \\
\hline Family history of affective disorder & 4920 & 24 & 1.00 & 0.45 & 2.24 & 0.99 & 1.01 & 0.45 & 2.26 & 0.98 \\
\hline Early onset of psychiatric problems ( $<18$ years) & 4191 & 20 & 1.65 & 0.68 & 3.98 & 0.27 & 1.78 & 0.66 & 4.80 & 0.26 \\
\hline Any comorbid psychiatric disorder & 7355 & 31 & 2.52 & 1.24 & 5.09 & 0.01 & 2.80 & 1.34 & 5.88 & 0.01 \\
\hline Comorbid substance use disorder & 7355 & 31 & 2.70 & 0.82 & 8.88 & 0.10 & 2.72 & 0.83 & 8.97 & 0.10 \\
\hline Comorbid anxiety disorder & 7355 & 31 & 1.84 & 0.75 & 4.49 & 0.18 & 1.89 & 0.77 & 4.66 & 0.17 \\
\hline Comorbid eating disorder & 7355 & 31 & 2.25 & 0.54 & 9.45 & 0.27 & 2.39 & 0.55 & 10.38 & 0.24 \\
\hline Comorbid personality disorder & 7355 & 31 & 4.37 & 1.68 & 11.39 & $<0.01$ & 4.78 & 1.77 & 12.89 & $<0.01$ \\
\hline Previous suicide attempt & 7328 & 31 & 3.71 & 1.75 & 7.88 & $<0.01$ & 4.31 & 1.91 & 9.71 & $<0.01$ \\
\hline Psychiatric inpatient care & 6268 & 25 & 2.51 & 1.05 & 6.02 & 0.04 & 2.50 & 1.04 & 6.01 & 0.04 \\
\hline Involuntary commitment & 5772 & 23 & 2.07 & 0.49 & 8.83 & 0.33 & 2.08 & 0.49 & 8.94 & 0.32 \\
\hline
\end{tabular}

HR, hazard ratio; BMI, body mass index; NOS, not otherwise specified; SADB, schizoaffective order, bipolar (manic) type.

${ }^{*}$ Adjusted for age.

could be an indicator of the severity of the disorder, was associated with suicide in our study. This finding also conveys an important clinical message as it may indicate that patients were discharged prematurely and/or without appropriate follow up. More specifically, we found a significant association between suicide and involuntary commitment, but it was only statistically significant in men when stratified on sex. Previous studies have not detected any association between suicide and a history of compulsory admissions (7), which could be due to the low number of individuals with bipolar disorder dying by suicide in those studies $(25,31)$.

In addition to the general risk factors for suicide above, there are also potential diagnosis-specific risk factors for suicide in bipolar disorder. These include affective episodes and subtype of the bipolar disorder. We found that recent affective episodes, especially depressive episodes, were significant predictors of suicide. This is in line with previous studies suggesting that depressive (17) but not manic (32) episodes are associated with increased likelihood of suicide in bipolar disorder. A Finnish study showed that $79 \%$ of suicides in bipolar disorder occurred during a major depressive episode, $11 \%$ during a mixed state, and $11 \%$ during or immediately after remission of psychotic mania (20). This is in line with the negative appraisals during the depressed state, which is a key element in the emergence of suicide ideation, according to the bipolar suicidality model (32). Importantly, the bipolar subtype was not associated with risk of suicide in the present study.

We have previously examined risk factors for suicide attempts in individuals with bipolar disorder (8). Several risk factors are shared between attempted and completed suicide: previous suicide attempts, comorbid anxiety disorder, recent affective episodes, and recent psychiatric inpatient care. In men, comorbid substance use disorder is a risk factor for both attempted and completed suicide. In women, comorbid personality disorder is a risk factor for both attempted and completed suicide. But we also discern factors associated with attempted but not with completed suicide, such as early onset of psychiatric problems, comorbid eating disorder, psychosocial and environmental problem/s, and a history of violent behavior.

Knowledge of risk factors for suicide is instrumental for clinicians who care for bipolar disorder patients. It is therefore important clinical 


\section{Hansson et al.}

knowledge that bipolar disorder patients - in addition to sharing risk factors for suicide in general have heightened risk after an affective/depressive episode. However, the limitations in relying on risk factors in preventing suicide should also be acknowledged.

\section{Strengths and limitations}

The major strengths of the current study are the prospective design and the large and representative sample: Both inpatients and outpatients with varying illness severity are included in this nation-wide cohort. Moreover, the quality register BipoläR contains more fine-grained phenotypic and demographic data than other Swedish national population registers. Finally, the Cause of Death Register captures $99 \%$ of all deaths in Sweden and it is unlikely that we have missed any suicides.

A limitation of the quality register is that it does not contain all patients with bipolar disorder in Sweden. According to the BipoläR annual report, the national coverage is $29 \%$ (33). As registration is voluntary both for participating clinics and patients, we cannot control for potential unknown inclusion bias and the possibility that some patient groups might be more likely to opt out from the quality register. But comparisons between the quality register and the national patient register (covering all patients with bipolar disorder in Sweden) have shown that the BipoläR cohort do not differ from the national cohort in terms of sex and age (33). In addition, several questions differed in the number of respondents due to two different versions of the questionnaire. This led to a drop in the number of respondents for some questions. Another limitation is that the results of this study performed in Sweden may not be generalizable to other countries. Finally, there are other potential risk factors for suicide that are not captured in the quality register BipoläR, such as early life adversities, family history of suicide, physical comorbidity, polarity of first episode, total number of lifetime episodes, and psychotic features in the depressive or manic episodes $(9,17,32,34)$.

\section{Acknowledgements}

This research was supported by grants from the Swedish Research Council (K2014-62X-14647-12-51), the Swedish foundation for Strategic Research (KF10-0039), the Swedish Brain Foundation, the Swedish Society for Medical Research, and the Swedish Federal Government under the LUA/ALF agreement (ALFGBG-142041).

Mathias Kardell is acknowledged for statistical and database support. We finally wish to thank the Swedish National Quality Register for Bipolar Affective Disorder: BipoläR.

\section{Declaration of interest}

None.

\section{References}

1. World Health Organization. Preventing suicide: a global imperative; 2014.

2. Jiang G, Hadlaczky G, Wasserman D. Självmord i Sverige, Data: 1980-2012, (NASP, ed); 2012.

3. Cavanagh JT, Carson AJ, Sharpe M, Lawrie SM. Psychological autopsy studies of suicide: a systematic review. Psychol Med 2003;33:395-405.

4. Pompili M, Gonda X, Serafini G et al. Epidemiology of suicide in bipolar disorders: a systematic review of the literature. Bipolar Disord 2013;15:457-490.

5. Chesney E, Goodwin GM, Fazel S. Risks of all-cause and suicide mortality in mental disorders: a meta-review. World Psychiatry 2014;13:153-160.

6. Osby U, Brandt L, Correia N, Ekbom A, Sparen P. Excess mortality in bipolar and unipolar disorder in Sweden. Arch Gen Psychiatry 2001;58:844-850.

7. Hawton K, Sutton L, Haw C, Sinclair J, Harriss L. Suicide and attempted suicide in bipolar disorder: a systematic review of risk factors. J Clin Psychiatry 2005;66:693-704.

8. Tidemalm D, Haglund A, Karanti A, Landen M, Runeson B. Attempted suicide in bipolar disorder: risk factors in a cohort of 6086 patients. PLoS One 2014;9:e94097.

9. Schaffer A, Isometsa ET, Tondo L et al. International Society for Bipolar Disorders Task Force on Suicide: metaanalyses and meta-regression of correlates of suicide attempts and suicide deaths in bipolar disorder. Bipolar Disord 2015;17:1-16.

10. Emilsson L, Lindahl B, Koster M, Lambe M, Ludvigsson JF. Review of 103 Swedish healthcare quality registries. J Intern Med 2015;277:94-136.

11. Sellgren C, Landen M, Lichtenstein P, Hultman CM, LANGSTRom N. Validity of bipolar disorder hospital discharge diagnoses: file review and multiple register linkage in Sweden. Acta Psychiatr Scand 2011;124:447-453.

12. National Board of Health and Welfare. Causes of Death 2010; 2011.

13. Runeson B, Tidemalm D, Dahlin M, Lichtenstein P, Langstrom N. Method of attempted suicide as predictor of subsequent successful suicide: national long term cohort study. BMJ 2010;341:c3222.

14. Neeleman J, Wessely S. Changes in classification of suicide in England and Wales: time trends and associations with coroners' professional backgrounds. Psychol Med 1997;27:467-472.

15. Therneau T, Crowson C, Atkinson E. Using time dependent covariates and time dependent coefficients in the Cox model; 2017.

16. Turecki G, Brent DA. Suicide and suicidal behaviour. Lancet 2016;387:1227-1239.

17. Schaffer A, Isometsa ET, Azorin JM et al. A review of factors associated with greater likelihood of suicide attempts and suicide deaths in bipolar disorder: part II of a report of the International Society for Bipolar Disorders Task Force on Suicide in Bipolar Disorder. Aust N Z J Psychiatry 2015;49:1006-1020.

18. Yoon YH, Chen CM, Yi HY, Moss HB. Effect of comorbid alcohol and drug use disorders on premature death among unipolar and bipolar disorder decedents in the United States, 1999 to 2006. Compr Psychiatry 2011;52:453-464.

19. Dennehy EB, Marangell LB, Allen MH, Chessick C, Wisniewski SR, Thase ME. Suicide and suicide attempts in 


\section{Risk factors for suicide in bipolar disorder}

the Systematic Treatment Enhancement Program for Bipolar Disorder (STEP-BD). J Affect Disord 2011;133: 423-427.

20. Isometsa ET, Henriksson MM, Aro HM, Lonnevist JK. Suicide in bipolar disorder in Finland. Am J Psychiatry 1994:151:1020-1024.

21. Clements C, Morriss R, Jones S, Peters S, Roberts C, Kapur $\mathrm{N}$. Suicide in bipolar disorder in a national English sample, 1996-2009: frequency, trends and characteristics. Psychol Med 2013;43:2593-2602.

22. Tidemalm D, Langstrom N, Lichtenstein P, Runeson B. Risk of suicide after suicide attempt according to coexisting psychiatric disorder: Swedish cohort study with long term follow-up. BMJ 2008;337:a2205.

23. Goldberg D, Fawcett J. The importance of anxiety in both major depression and bipolar disorder. Depress Anxiety 2012;29:471-478.

24. Simon Ge, Hunkeler E, Fireman B, Lee JY, Savarino J. Risk of suicide attempt and suicide death in patients treated for bipolar disorder. Bipolar Disord 2007;9:526-530.

25. King EA, Baldwin DS, Sinclair JM, BaKer NG, Campbell MJ, Thompson C. The Wessex recent in-patient suicide study, 1. Case-control study of 234 recently discharged psychiatric patient suicides. Br J Psychiatry 2001;178:531536.

26. Boardman AP, Grimbaldeston AH, Handley C, Jones PW, Willmott S. The North Staffordshire Suicide Study: a case-control study of suicide in one health district. Psychol Med 1999;29:27-33.

27. Stenbacka M, Romelsjo A, Jokinen J. Criminality and suicide: a longitudinal Swedish cohort study. BMJ Open 2014;4:e03497.
28. Hawton K, van Heeringen K. Suicide. Lancet 2009;373: $1372-1381$.

29. Fazel S, Ramesh T, Hawton K. Suicide in prisons: an international study of prevalence and contributory factors. Lancet Psychiatry 2017;4:946-952.

30. Haglund A, Tidemalm D, Jokinen $\mathbf{J}$ et al. Suicide after release from prison: a population-based cohort study from Sweden. J Clin Psychiatry 2014;75:1047-1053.

31. Steblaj A, Tavcar R, Dernovsek MZ. Predictors of suicide in psychiatric hospital. Acta Psychiatr Scand 1999; 100:383-388

32. Malhi GS, Outhred T, Das P, Morris G, Hamilton A, ManNIE Z. Modeling suicide in bipolar disorders. Bipolar Disord 2018;20:334-348.

33. PÅlsson E, LAndÉn M. Kvalitetsregister BipoläR Årsrapport 2016 (Swedish) [The quality register BipoläR annual report 2016]. https://bipolar.registercentrum.se/om-bipola r/arsrapporter/p/SkMRS-nGb; 2017.

34. Agnew-Blais J, Danese A. Childhood maltreatment and unfavourable clinical outcomes in bipolar disorder: a systematic review and meta-analysis. Lancet Psychiatry 2016;3:342-349.

\section{Supporting Information}

Additional supporting information may be found online in the Supporting Information section at the end of the article:

Table S1. Number (\%) of patients having each potential risk factor at least once during the study period. 